\title{
Two-Dimensional FDTD Inverse-Scattering Scheme for Determination of Near-Surface Material Properties at Microwave Frequencies
}

\author{
Milica Popović, Member, IEEE, and Allen Taflove, Fellow, IEEE
}

\begin{abstract}
This paper reports a two-dimensional time-domain inverse-scattering algorithm based upon the finite-difference timedomain method for determining near-surface material properties at microwave frequencies. The time-domain nature of this technique allows for limiting the region of inversion using causality. The algorithm traces a search trajectory in the $\left(\varepsilon_{r}, \sigma\right)$ parameter space. The minimal parameter estimation error along this trajectory yields a set of approximate parameter values. It is shown that the algorithm's convergence and robustness with respect to broadband Gaussian noise depends on the shape and the duration of the illuminating electromagnetic wave pulse chosen for the electrical parameter reconstruction. It is possible to achieve an unambiguous indication of algorithm convergence despite the noisy background.
\end{abstract}

Index Terms-Finite-difference time-domain (FDTD), inverse scattering, two-dimensional (2-D).

\section{INTRODUCTION}

$\mathbf{N}$ UMERICAL inverse-scattering studies found in the literature are based on either frequency- or time-domain approaches. With frequency-domain algorithms, the interaction of the entire medium with the incident field is considered simultaneously [1]-[4]. For a general, lossy inhomogeneous medium this can result in a very large number of unknown parameters. In contrast, time-domain approaches can exploit causality to limit the region of inversion, potentially reducing the number of unknowns.

Time-domain inverse-scattering problems somewhat related to the present study commonly appear in the area of geoscience and remote sensing [5]-[7]. In such problems, ground-penetrating radar (GPR) is used to obtain information on subsurface features from data collected over the surface. Signal-processing algorithms, including those based on finite-difference time-domain (FDTD) [5], [6], can be used for target reconstruction. Global inversion techniques have emerged involving the neural network method [6] and the use of genetic algorithms [7]. These algorithms, based on stochastic strategies, offer advantages relative to local inversion algorithms including strong search ability,

Manuscript received August 28, 2001; revised January 10, 2003. This work was supported by the U.S. Department of Defense (DOD) Predoctoral Grant DAMD17-99-1-9335. Computing resources were provided by Cray Inc.

M. Popović is with the Department of Electrical and Computer Engineering, McGill University, Montreal, QC H3A-2A7, Canada (e-mail: poppy@ece.mcgill.ca).

A. Taflove is with the Department of Electrical and Computer Engineering, McCormick School of Engineering, Northwestern University, Evanston, IL 60208 USA.

Digital Object Identifier 10.1109/TAP.2004.832515 simplicity, robustness, and insensitivity to ill-posedness. However, they require a large number of function evaluations.

More relevant to the present work are the FDTD/nonlinear optimization techniques of [8] and [9]. [8] reported a one-dimensional (1-D) FDTD formulation of an inverse-scattering scheme for remote sensing of inhomogeneous lossy layered media. Here, a layer-stripping procedure was used to simultaneously recover the conductivity and permittivity profiles. This work assumed a normally incident plane wave pulse and zero noise present on the received pulse.

[9] reported a two-dimensional (2-D) FDTD inverse-scattering scheme for remote sensing of the shape of conducting and dielectric targets. With this technique, as the illuminating wavefront sweeps across the target, causality is exploited to reconstruct the actual target surface contour in a sequential and cumulative manner. To test the complexity of a recoverable structure, a plane-wave-illuminated dielectric target with reentrant features was reconstructed from a single-point observation. Numerical experiments tested the performance of the algorithm in the presence of noise and demonstrated a promising degree of robustness.

The present work focuses on the development of a time-domain inverse-scattering algorithm that permits a noninvasive measurement of the near-surface dielectric parameters of a lossy layered half-space. The new algorithm extends the iterative technique of [8] to the case of a 2-D half-space impulsively excited at its surface by an infinitely long monopole. Both the time waveform and the duration of the exciting pulse are studied to explore optimization of the convergence of the inverse-scattering technique. Further, the robustness of the technique in the presence of noise is examined.

We choose to illustrate our new time-domain inverse-scattering algorithm in the context of measurement of the dielectric parameters and skin thickness of the female human breast. For breast skin tissue, the permittivity and conductivity values found in the literature are approximately $\varepsilon_{r-\text { skin }}=36$ and $\sigma_{\text {skin }}=\mathrm{S} / \mathrm{m}$ in the microwave frequency range [10]-[13]. The literature offers several studies, for example [14], [15], indicating that breast skin thickness ranges from $0.7-2.7 \mathrm{~mm}$ depending upon the location. The reported causes of mammary skin thickening are commonly categorized by their local or generalized nature. The localized causes can be carcinoma, inflammation, trauma, fat necrosis, postbiopsy, and dermatological conditions. The generalized causes are breast cancer, Hodgkin's disease, reticulum cell sarcoma, metastatic disease, radiation therapy, inflammation, surgery, primary skin disorders, anasarca and any cause of 
lymphatic obstruction. Therefore, careful monitoring of nearsurface breast properties can be used as a prediagnostic tool of the underlying tissue pathologies or health concerns of the patient in general.

This paper first reviews the single-parameter reconstruction of skin dielectric properties using the proposed time-domain inverse-scattering algorithm. Next, results are presented to show the development of a 2-D time-domain inverse-scattering algorithm for simultaneous estimation of electrical permittivity $\varepsilon_{r-\text { skin }}$ and conductivity $\sigma_{\text {skin }}$ of the skin layer. This algorithm locates a search trajectory in the $\left(\varepsilon_{r-\text { skin }}, \sigma_{\text {skin }}\right)$ parameter space. Results are then presented which show that the convergence of the search trajectory and the convergence error depends on the shape and the duration of the impinging pulse. This convergence then permits the skin thickness to be determined by comparing the measurement with a simulated all-skin response. Finally, the robustness of the algorithm is tested in the presence of Gaussian noise for various signal-to-noise ratios.

\section{Single-PARAMETER ReCONSTRUCTION OF SKIN DIELECTRIC PROPERTIES}

This section introduces the basic time-domain inverse-scattering technique by considering the case of single-parameter reconstruction of a skin layer over a homogeneous lossy halfspace. We assume knowledge of the skin thickness. The principal logic of this scheme is shown in Fig. 1.

Based on an initial guess for a set of dielectric parameters $\left(\varepsilon_{r-\text { skin }}, \sigma_{\text {skin }}\right)$, the FDTD code computes a trial time-waveform for the magnetic field immediately adjacent to the monopole during a time window $T_{\text {window }}$. $T_{\text {window }}$ is selected based upon the assumed knowledge of the skin thickness to be short enough to avoid contamination of the trial waveform by reflections from the interface between the skin and the underlying half-space. At each time-step during this run, we calculate the square of the difference between the trial and measured magnetic field waveforms at this point, and add this to a running sum which constitutes the energy-normed error $U$. This value is used to perturb the dielectric parameter under investigation in the following manner:

1. Keeping the other dielectric parameter fixed, the dielectric parameter under investigation is incremented by an arbitrary fixed amount $\Delta$, where $\Delta$ can be either positive or negative.

2. The FDTD code is rerun, and a new value of $U$ is calculated.

3. If the new value of $U$ is less than the previous value, the dielectric parameter under investigation is again incremented by $\Delta$. However, if the new value of $U$ is greater than the previous value, the dielectric parameter under investigation is incremented by $-\Delta / 2$.

There are two ways to exit the iterative loop of Fig. 1. For exit $1, U$ decreases below a predetermined threshold value. For

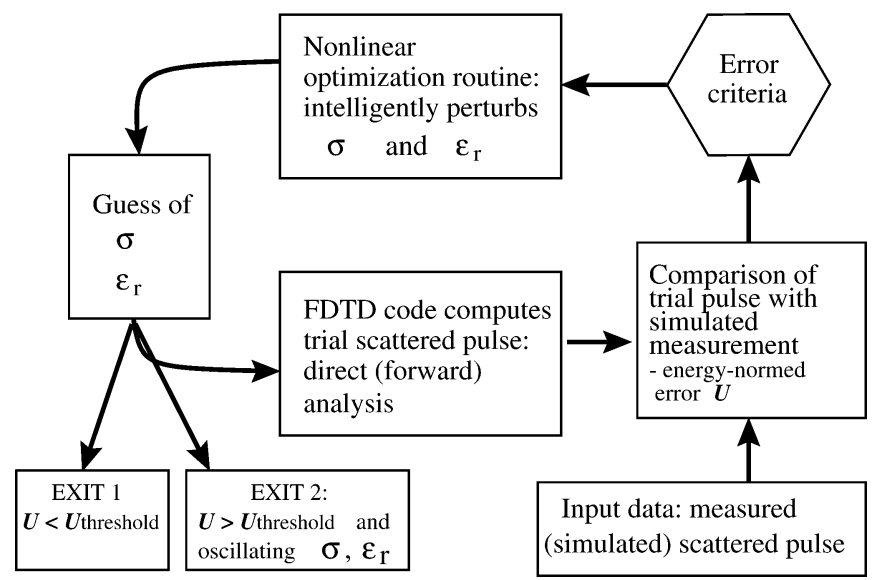

Fig. 1. Time-domain inverse-scattering iterative algorithm for recovery of dielectric parameters $\varepsilon_{r}$ and $\sigma$ from a measured (in this study, simulated measured) signal. The scheme exploits causality to limit the region of inversion.

exit 2, $U$ remains above the threshold but the estimated $\varepsilon_{r}$ and $\sigma$ parameters oscillate within a narrow band of values a predetermined number of times. Both of these exit criteria are chosen according to physical meaningfulness and to limitations imposed by available computational resources.

Here, we illustrate the convergence properties of the timedomain inverse-scattering algorithm of Fig. 1 for a skin halfspace with only one unknown parameter, $\varepsilon_{r-\text { skin }}$ or $\sigma_{\text {skin. }}$. A 120 -ps (1/e full-width) differentiated Gaussian pulse is used as the excitation waveform.

In Case 1 [Fig. 2(a)], we fix the value of $\sigma_{\text {skin }}$ at the correct value of $4 \mathrm{~S} / \mathrm{m}$ while iterating for $\varepsilon_{r \text {-skin }}$. Here, the FDTD code computes the backscattered signal for a trial value of $\varepsilon_{r-\text { skin }}$, compares it with the reference backscattered signal obtained for the correct value of $\varepsilon_{r-\text { skin }}$, and calculates the energy-normed error. Based on this error, $\varepsilon_{r \text {-skin }}$ is perturbed, and the FDTD code computes the backscattered signal for this new guess for the permittivity. This is repeated until one of the criteria for exiting the iterative process is met. Sample results are shown for two initial guesses of $\varepsilon_{r-s k i n}, 99$ and 150 .

In Case 2 [Fig. 2(b)], we fix the value of $\varepsilon_{r-\text { skin }}$ at the correct value of 36 while iterating for $\sigma_{\text {skin. }}$. Sample results are shown for two initial guesses for $\sigma_{\text {skin }}, 19 \mathrm{~S} / \mathrm{m}$ and $20 \mathrm{~S} / \mathrm{m}$. In these and similar simulations, we have found a robust convergence for $\varepsilon_{r-\text { skin }}$ and $\sigma_{\text {skin }}$ in the absence of noise for a variety of parameter perturbation increments.

\section{Two-PARAMETER ReCONSTRUCTION OF SKIN DIELECTRIC PROPERTIES}

\section{A. Methods}

This section describes an optimization scheme which allows simultaneous recovery of the permittivity and conductivity of the skin. This scheme generates a search trajectory in the $\left(\varepsilon_{r-s k i n}, \sigma_{\text {skin }}\right)$ space that ideally converges to the reference values of these parameters.

The search trajectory is generated in two possible ways.

1) A trial value of $\sigma_{\text {skin }}$ is assumed and a series of FDTD forward-scattering runs is conducted to find a corresponding value of $\varepsilon_{r \text {-skin }}$ which minimizes the energy-normed error 


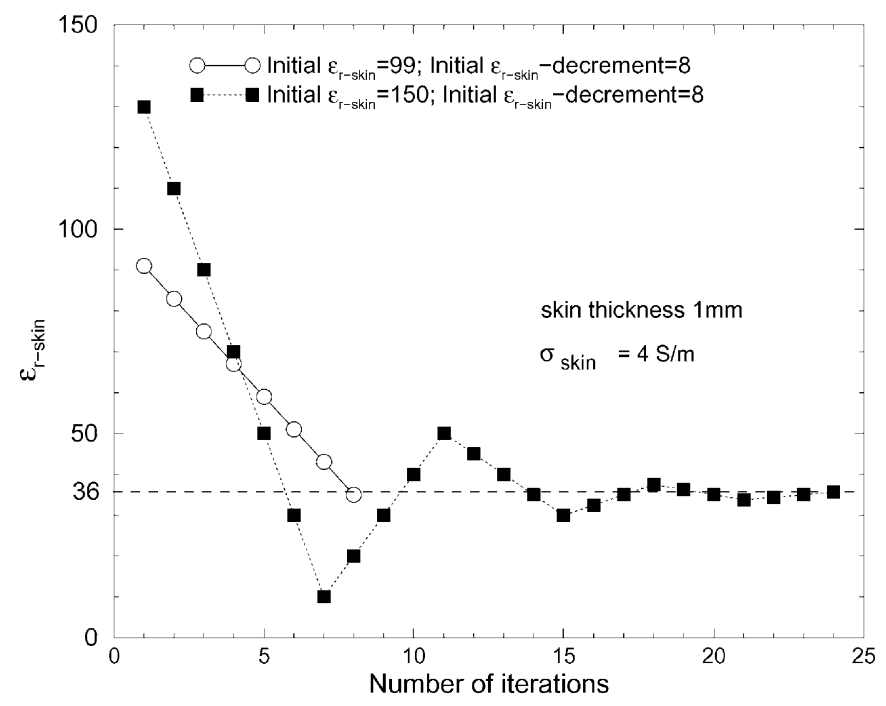

(a)

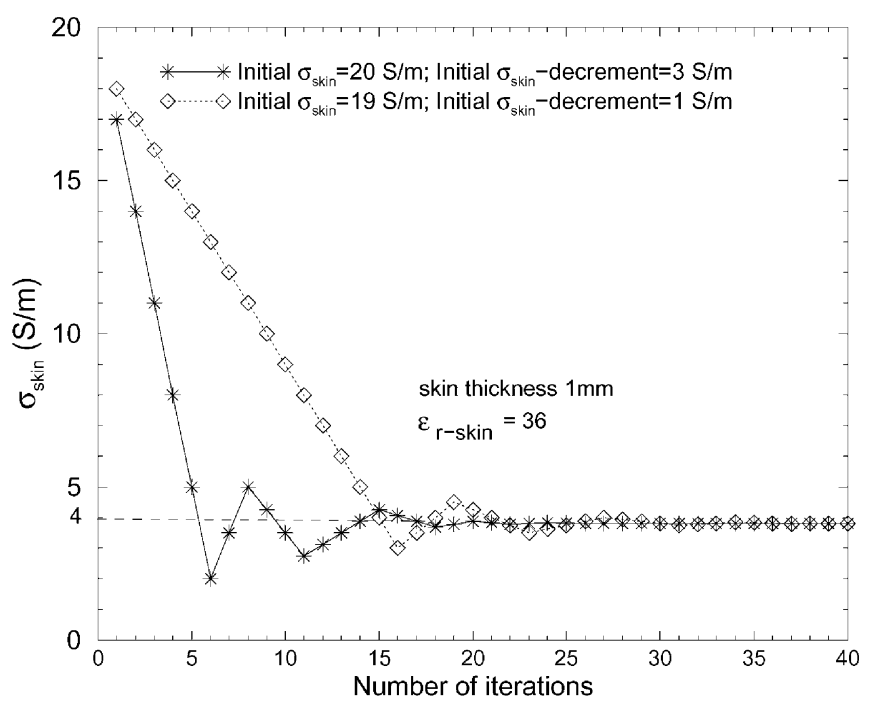

(b)

Fig. 2. Inverse-scattering FDTD computation for a skin half-space: (a) Convergence of $\varepsilon_{r \text {-skin }}$ to its correct value of 36 for two different initial guesses of $\varepsilon_{r \text {-skin }}$, with $\sigma_{\text {skin }}$ fixed at the correct value of $4 \mathrm{~S} / \mathrm{m}$. (b) Convergence of $\sigma_{\text {skin }}$ to its correct value of $4 \mathrm{~S} / \mathrm{m}$ for two different initial guesses of $\sigma_{\text {skin }}$, with $\varepsilon_{r \text {-skin }}$ fixed at the correct value of 36 .

relative to the measured backscattered waveform. This procedure is then repeated for a sequence of trial values of $\sigma_{\text {skin }}$ which vary uniformly in equal increments within a $\pm 50 \%$ range about the nominal value reported in the literature.

2) A trial value of $\varepsilon_{r \text {-skin }}$ is assumed and a series of FDTD forward-scattering runs is conducted to find a corresponding value of $\sigma_{\text {skin }}$ which minimizes the energy-normed error relative to the measured backscattered waveform. This procedure is then repeated for a sequence of trial values of $\varepsilon_{r-\text { skin }}$ which vary uniformly in equal increments within a \pm 50 range about the nominal value reported in the literature.

We here introduce the excitation waveforms used in our investigations. The results section will show that the search trajectory in the $\left(\varepsilon_{r-\text { skin }}, \sigma_{\text {skin }}\right)$ space depends strongly on the signal shape and duration of the excitation waveform. The three $E_{z}$-field excitation waveforms used for the dielectric parameter reconstruction are as follows: 1) 120-ps differentiated Gaussian pulse; 2) 10-ps differentiated Gaussian pulse with a 5-ps risetime; and 3) 5-ps rise time ramp which has the same maximum as the peak value of the 10-ps differentiated Gaussian pulse.

It is important to note the following. The inverse-scattering algorithm processes only a time window $T_{\text {window }}$ within which it compares the trial and measured signals. For breast-skin parameter recovery, it is critical that $T_{\text {window }}$ be small enough to avoid wave reflection from the underlying skin-breast interface which would corrupt the received signal. (In practical situations, the selection of $T_{\text {window }}$ would be conservatively based upon the minimum values of skin thickness and permittivity reported in the literature.) This means that only a part of the measured backscattered waveform might be usable for accurately generating a trajectory in the $\left(\varepsilon_{r-\text { skin }}, \sigma_{\text {skin }}\right)$ space. For example, in the next section, we will see that, for the 120-ps differentiated Gaussian pulse excitation case, only the leading edge of the measured backscattered pulse is used in the reconstruction. Conversely, the entire measured backscattered pulse is used for the 10-ps differentiated Gaussian pulse excitation case. Results will illustrate the significant consequences that this has on the calculated trajectory in the $\left(\varepsilon_{r-\text { skin }}, \sigma_{\text {skin }}\right)$ space.

\section{B. Results in the Absence of Noise}

1) $\varepsilon_{r-s k i n}$ Reconstruction With Trial Values of $\sigma_{\text {skin }}$ : This section reports results for the inverse-scattering algorithm iterating for $\varepsilon_{r \text {-skin }}$ using a range of trial values of $\sigma_{\text {skin }}$ varying \pm $50 \%$ around the reference value of $\sigma_{\text {skin }}=4 \mathrm{~S} / \mathrm{m}$. In all simulations, the time step used in the FDTD code is $\Delta t=0.33356$ ps.

Fig. 3(a) depicts the resulting trajectory in the $\left(\varepsilon_{r-\text { skin }}, \sigma_{\text {skin }}\right)$ space for the 120-ps differentiated Gaussian pulse excitation and $T_{\text {window }}=400 \Delta t$. This trajectory passes through the reference value $\varepsilon_{r-\text { skin }}=36$. Fig. 3(a) also shows the large error made in the $\varepsilon_{r \text {-skin }}$ recovery if $T_{\text {window }}=600 \Delta t$, which is large enough to include the unwanted reflection from the underlying skinbreast tissue interface. Fig. 3(b) graphs the error for $T_{\text {window }}$ for each value in the trial $\sigma_{\text {skin }}$ range for its corresponding estimated $\varepsilon_{r-\text { skin }}$ of Fig. 3(a). Fig. 3(b) shows that the error function for $\varepsilon_{r-\text { skin }}$ recovery using trial values of $\sigma_{\text {skin }}$ suffers from local minima, and thus cannot be used as a search criterion along the trajectory in the $\left(\varepsilon_{r-\text { skin }}, \sigma_{\text {skin }}\right)$ space. Similar results were observed for the 10-ps differentiated Gaussian pulse and 5-ps rise-time ramp excitation.

2) $\sigma_{\text {skin }}$ Reconstruction With Trial Values of $\varepsilon_{r-\text { skin: }}$ This section reports results for the inverse-scattering algorithm iterating for $\sigma_{\text {skin }}$ using a range of trial values of $\varepsilon_{r-\text { skin }}$ varying \pm $50 \%$ around the reference value of $\varepsilon_{r-\text { skin }}=36$. In all simulations, the time step used in the FDTD code is $\Delta t=0.3335$ ps.

Results for the excitation signal waveforms under investigation are shown in Figs. 4-6. For both the 120-ps differentiated Gaussian pulse (Fig. 4) and the 5-ps rise-time ramp (Fig. 5), $T_{\text {window }}$ includes only the leading edge of the backscattered pulse waveform. This window duration is conservatively chosen in the manner discussed previously to avoid contamination by wave reflections at the interface between the skin and the underlying tissue. This yields in Figs. 4(a) and 5(a) linear search 


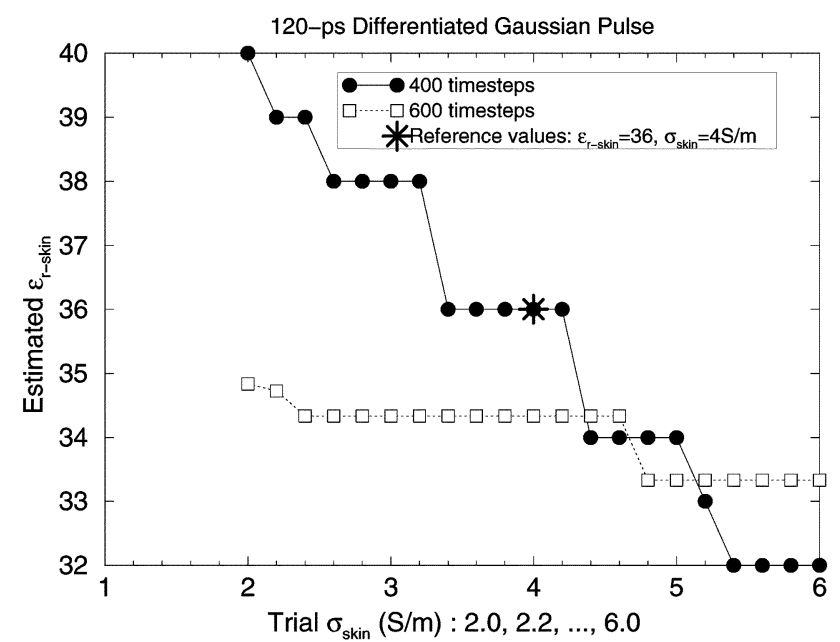

(a)

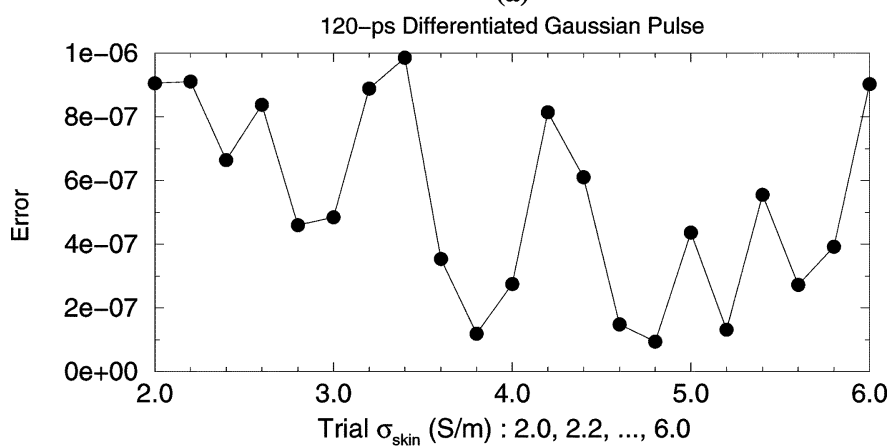

(b)

Fig. 3. Estimate of $\varepsilon_{r \text {-skin }}$ for trial values of $\sigma_{\text {skin }}$ for the 120-ps differentiated Gaussian pulse excitation case. (a) Search trajectory in $\left(\varepsilon_{r-\text { skin }}, \sigma_{\text {skin }}\right)$ space for $T_{\text {window }}=400 \Delta t$ and $T_{\text {window }}=600 \Delta t$, where $\Delta t=0.33356 \mathrm{ps}$. (b) Error versus trial values of $\sigma_{\text {skin }}$ for $T_{\text {window }}=400 \Delta t$.

trajectories in the $\left(\varepsilon_{r-s k i n}, \sigma_{\text {skin }}\right)$ space. For both of these excitations, Figs. 4(b) and 5(b) show a broad minimum of the error along the search trajectory as a function of the estimated $\sigma_{\text {skin }}$ value. This minimum is approximately centered at the location of the assumed reference values of $\varepsilon_{r \text {-skin }}$ and $\sigma_{\text {skin }}$, implying that minimizing the error could be used as a search criterion along the trajectory in the $\left(\varepsilon_{r-\text { skin }}, \sigma_{\text {skin }}\right)$ space. However, the broad error minimum suggests sensitivity of the skin dielectric parameter recovery in the presence of noise.

Fig. 6(a) graphs the search trajectory in the $\left(\varepsilon_{r-\text { skin }}, \sigma_{\text {skin }}\right)$ space obtained for the 10-ps differentiated Gaussian pulse excitation. Here, $T_{\text {window }}$ includes the entire backscattered pulse waveform, and the search trajectory shows a parabolic behavior. We note a sharp null of the error along the search trajectory at the location of the assumed reference values of the skin dielectric parameters, which suggests robustness of parameter recovery in the presence of noise. This is explored next.

\section{Results in the Presence of Zero-Mean Gaussian Noise}

We now test the robustness of the inverse-scattering scheme of the previous section by adding zero-mean Gaussian noise to the simulated measured backscattered signals. The signal-tonoise $(\mathrm{S} / \mathrm{N})$ ratio is defined by $20 \log _{10}\left(\operatorname{Signal}_{\mathrm{MAX}} /\right.$ Noise $\left._{\mathrm{SD}}\right)$,

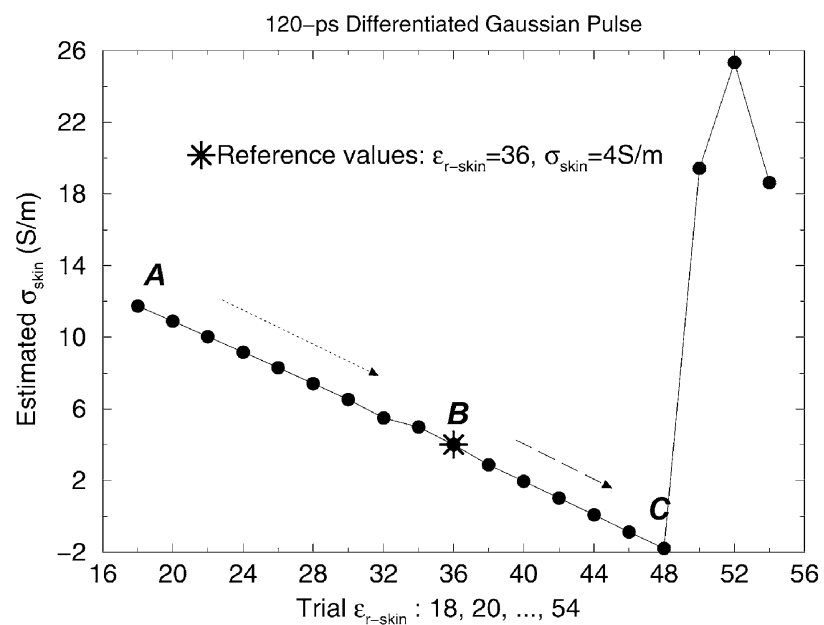

(a)

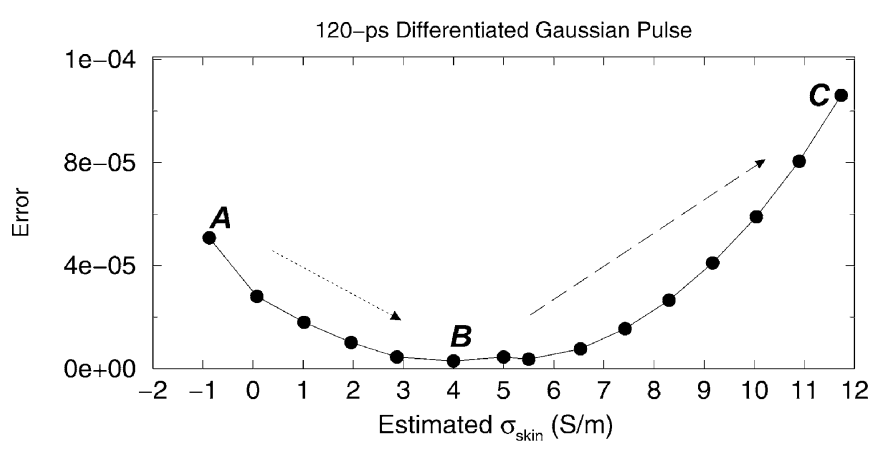

(b)

Fig. 4. Estimate of $\sigma_{\text {skin }}$ for trial values of $\varepsilon_{r-\text { skin }}$ for the 120-ps differentiated Gaussian pulse excitation. (a) Search trajectory in $\left(\varepsilon_{r-s k i n}\right.$, $\left.\sigma_{\text {skin }}\right)$ space for $T_{\text {window }}=400 \Delta t$, where $\Delta t=0.33356 \mathrm{ps}$. (b) Error versus estimated $\sigma_{\text {skin }}$ values, showing a broad null at the location of the assumed reference values of the skin parameters.

where Signal $_{\text {MAX }}$ is the peak value of the noiseless backscattered waveform and Noise $_{\mathrm{SD}}$ is the standard deviation of the zero-mean Gaussian noise.

This section presents results for noisy backscattered signals resulting from the 10-ps differentiated Gaussian pulse excitation. Individual search trajectories in the $\left(\varepsilon_{r-\text { skin }}, \sigma_{\text {skin }}\right)$ space are generated for five independent noise samples added to the backscattered signal for $\mathrm{S} / \mathrm{N}$ ratios of 20 and $40 \mathrm{~dB}$. Then, for each $\mathrm{S} / \mathrm{N}$ level, the five data sets are averaged before input to the inverse-scattering algorithm to yield a final search trajectory. The latter procedure simulates the desirable outcome of "boxcar integration" which is often used as a technique for measuring signals contaminated with zero-mean noise wherein multiple measurements are averaged to enhance the deterministic signal. For all results shown, $T_{\text {window }}=80 \Delta t$, where $\Delta t=$ 0.33356 ps. Figs. 7(a) and 8(a) show the generated search trajectories in the $\left(\varepsilon_{r-\text { skin }}, \sigma_{\text {skin }}\right)$ space for each noisy waveform and for the averaged backscattered waveform for $\mathrm{S} / \mathrm{N}=20$ and $40 \mathrm{~dB}$, respectively. As expected, for the higher S/N ratio, we observe a smaller deviation of the individual trajectories from the trajectory obtained using the averaged backscattered signal.

More importantly Figs. 7(b) and 8(b) show that the corresponding error along the search trajectory as a function of the estimated $\sigma_{\text {skin }}$ exhibits a sharp null at the correct value of 


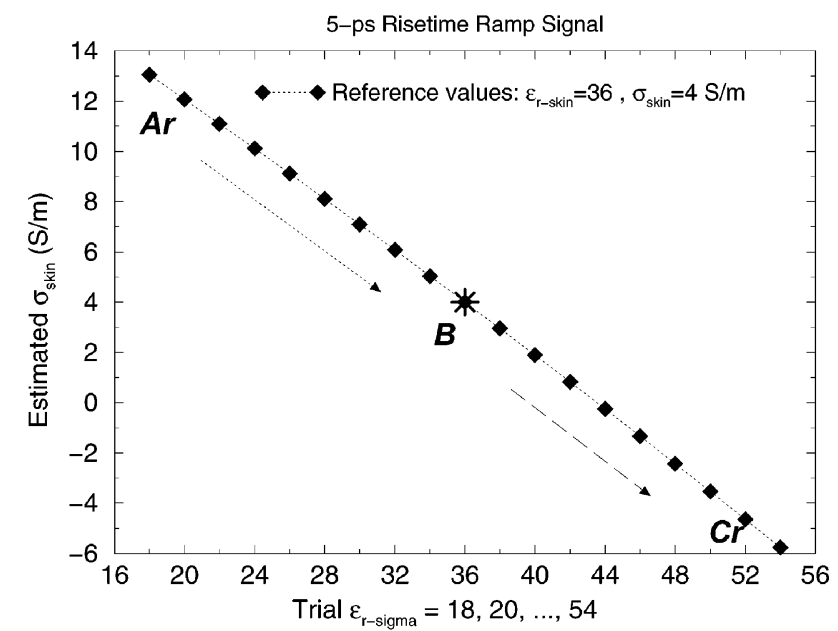

(a)

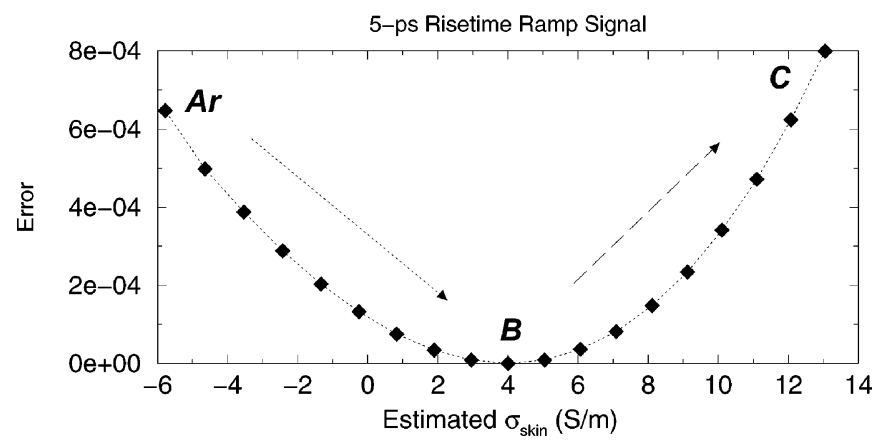

(b)

Fig. 5. Estimate of $\sigma_{\text {skin }}$ for trial values of $\varepsilon_{r-\text { skin }}$ for the 5-ps rise-time ramp excitation. (a) Search trajectory in $\left(\varepsilon_{r-\text { skin }}, \sigma_{\text {skin }}\right)$ space for $T_{\text {window }}=$ $100 \Delta t$, where $\Delta t=0.33356 \mathrm{ps}$. (b) Error versus estimated $\sigma_{\text {skin }}$ values, showing a broad null at the location of the assumed reference values of the skin parameters.

$\varepsilon_{r-\text { skin }}=36$, even for the lower value of $\mathrm{S} / \mathrm{N}$. This unambiguous indication of the correct value of $\varepsilon_{r-\text { skin }}$ despite a noisy background is crucial in the determination of the skin thickness, to be discussed next.

\section{DETERMINING SKIN THICKNESS}

We now briefly describe how we can estimate the breast skin thickness once the patient-specific value for $\varepsilon_{r \text {-skin }}$ is determined. Note that this procedure is relatively insensitive to $\sigma_{\text {skin }}$. Here, the excitation signal shape and duration is much less critical than before.

Due to the dielectric contrast between the skin and the underlying breast tissue, the propagating pulse reflects off the skin-breast tissue interface. By comparing the measured response for the finite skin-thickness case with an FDTD simulation which assumes a homogeneous skin half-space characterized by the $\varepsilon_{r \text {-skin }}$ determined previously, the observed time of the first reflection yields the data required to determine the skin thickness.

To illustrate this strategy, Fig. 9 graphs the results of four test cases for an assumed 60-ps differentiated Gaussian pulse excitation wherein measured backscattered waveforms for skin thicknesses of $0.6,1.2,1.8$ and $2.4 \mathrm{~mm}$ and $\varepsilon_{r-\text { skin }}=36$ are simulated using 2-D FDTD models. These waveforms are subtracted

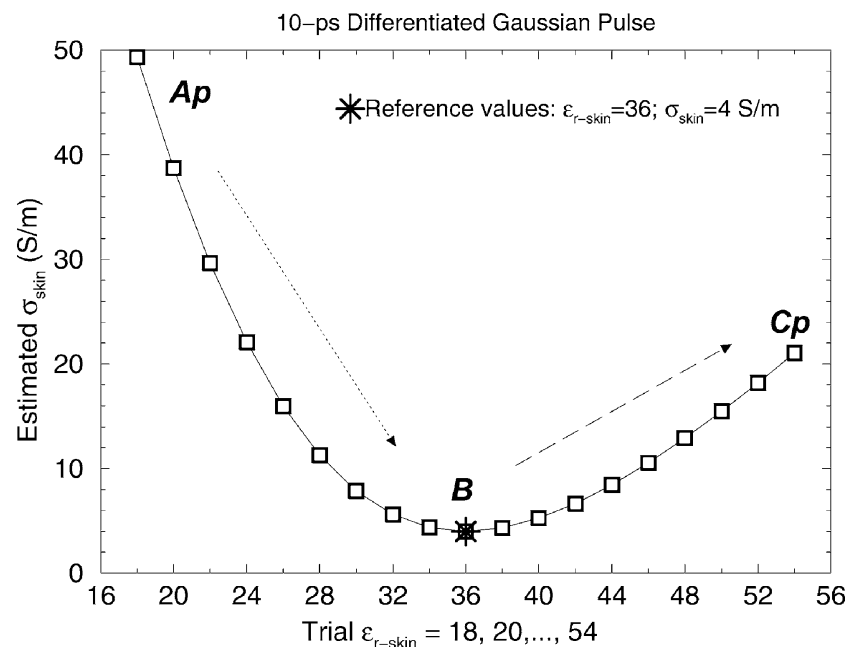

(a)

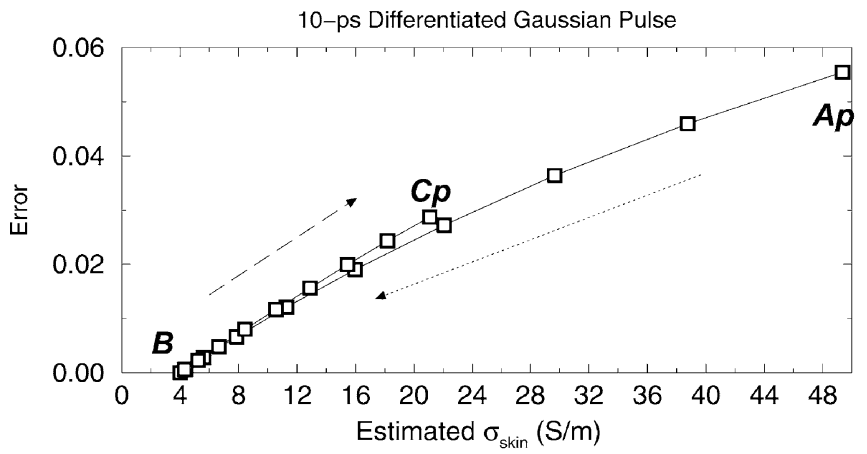

(b)

Fig. 6. Estimate of $\sigma_{\text {skin }}$ for trial values of $\varepsilon_{r-\text { skin }}$ for the 10-ps differentiated Gaussian pulse excitation. (a) Search trajectory in $\left(\varepsilon_{r-\text { skin }}, \sigma_{\text {skin }}\right)$ space for $T_{\text {window }}=80 \Delta t$, where $\Delta t=0.33356 \mathrm{ps}$. (b) Error versus estimated $\sigma_{\text {skin }}$ values, showing a sharp null at the location of the assumed reference values of the skin parameters.

from the FDTD-calculated backscattered response for the corresponding skin half-space. Then, the estimated time delay of the peak value of the difference signal with respect to the peak of the skin half-space response is used to estimate the skin thickness. The resulting breast skin thickness values obtained in this manner are $0.51,1.25,1.85$ and $2.45 \mathrm{~mm}$, respectively.

\section{DISCUSSION}

\section{A. Impact of the Excitation Signal Shape and Duration Upon the Robustness of the Inverse-Scattering Algorithm}

The results of the previous sections show that the robustness of the time-domain inverse-scattering algorithm for recovery of $\varepsilon_{r-\text { skin }}$ and $\sigma_{\text {skin }}$ depends on the duration and shape of the excitation waveform. Specifically, of the excitations considered, the 10-ps differentiated Gaussian pulse provides superior robustness, even relative to the ramp signal having the same rise-time, if $T_{\text {window }}$ includes the entire backscattered waveform due to the differentiated Gaussian pulse. Apparently, a bipolar excitation delivers more information to the proposed inverse-scattering algorithm about the signal-path propagation medium than a comparably fast unipolar excitation. The sharp error null consistently obtained along the search generated trajectory in the $\left(\varepsilon_{r-\text { skin }}, \sigma_{\text {skin }}\right)$ space for the 10 -ps differentiated 
$\mathrm{S} / \mathrm{N}=20 \mathrm{~dB}$

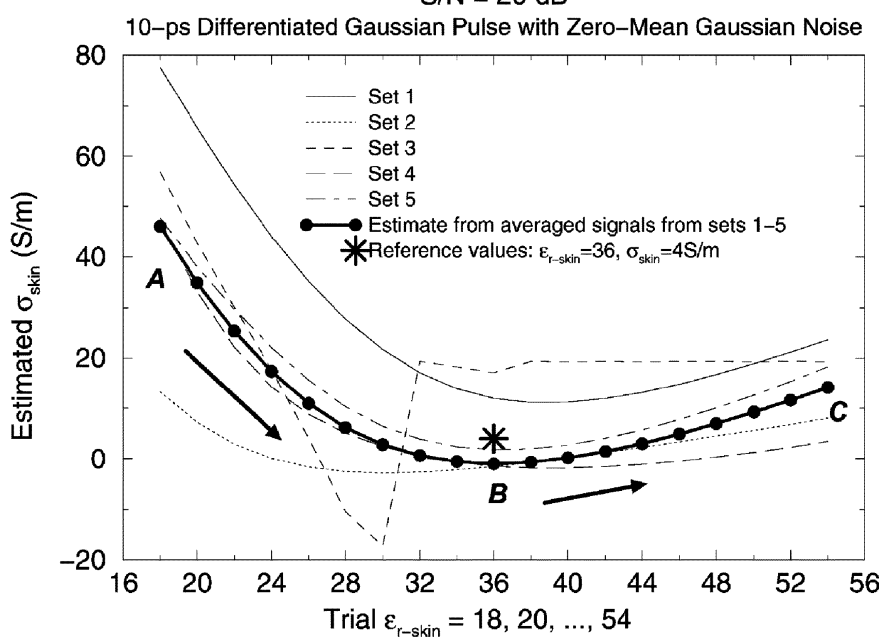

(a)

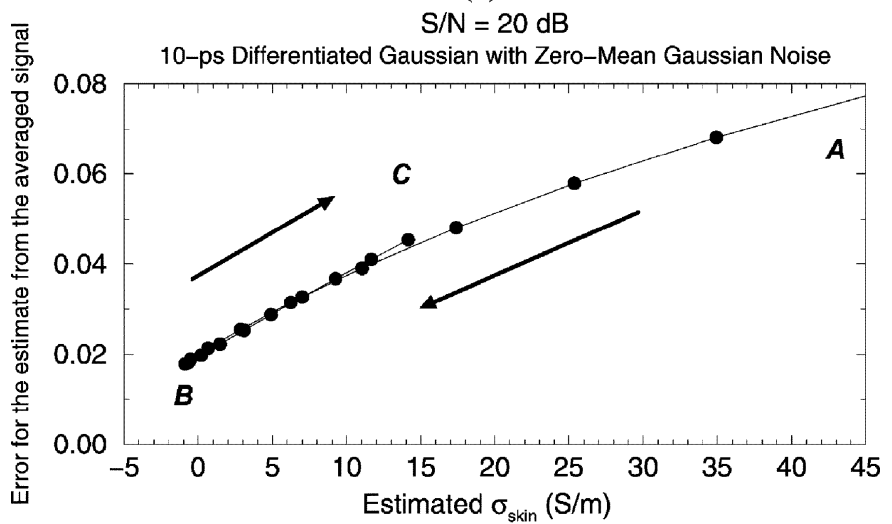

(b)

Fig. 7. (a) Sample trajectories in the $\left(\varepsilon_{r-s k i n}, \sigma_{\text {skin }}\right)$ space for the 10-ps differentiated Gaussian pulse excitation with $\mathrm{S} / \mathrm{N}=20 \mathrm{~dB}$. (b) Error versus estimated $\sigma_{\text {skin }}$ values for the averaged-backscattered-waveform case.

Gaussian pulse excitation, even in the presence of significant levels of zero-mean Gaussian noise, clearly illustrates this point. Signal-to-noise ratios as low as $20 \mathrm{~dB}$ are adequate for reliable recovery of the skin permittivity using this excitation.

\section{B. Linearity of Estimated $\sigma_{\text {skin }}$ Versus $\varepsilon_{r-\text { skin }}$ in the Case of Time-Linear Excitations}

In the previous sections, we observed that the search trajectory in the $\left(\varepsilon_{r-\text { skin }}, \sigma_{\text {skin }}\right)$ space is a straight line for cases where the leading edge of the 120-ps differentiated Gaussian pulse or the 5-ps rise-time ramp excitation was used for simulation of backscattered signals and parameter reconstruction. This was observed even in the presence of zero-mean Gaussian noise. In this section, we analyze the connection between the linear time-dependence of the excitation pulse and the linearity of the generated search trajectory.

We begin the analysis with Ampere's law in three dimensions for linear, isotropic, nondispersive, lossy materials

$$
\nabla \times \vec{H}=\left(\vec{J}_{\text {source }}+\sigma \vec{E}\right)+\varepsilon \cdot \frac{\partial \vec{E}}{\partial t} .
$$

Here, $\vec{H}$ is the magnetic field $(\mathrm{A} / \mathrm{m}) ; \vec{E}$ is the electric field $(\mathrm{V} / \mathrm{m}) ; \vec{J}_{\text {source }}$ is an independent source current density $\left(\mathrm{A} / \mathrm{m}^{2}\right)$;

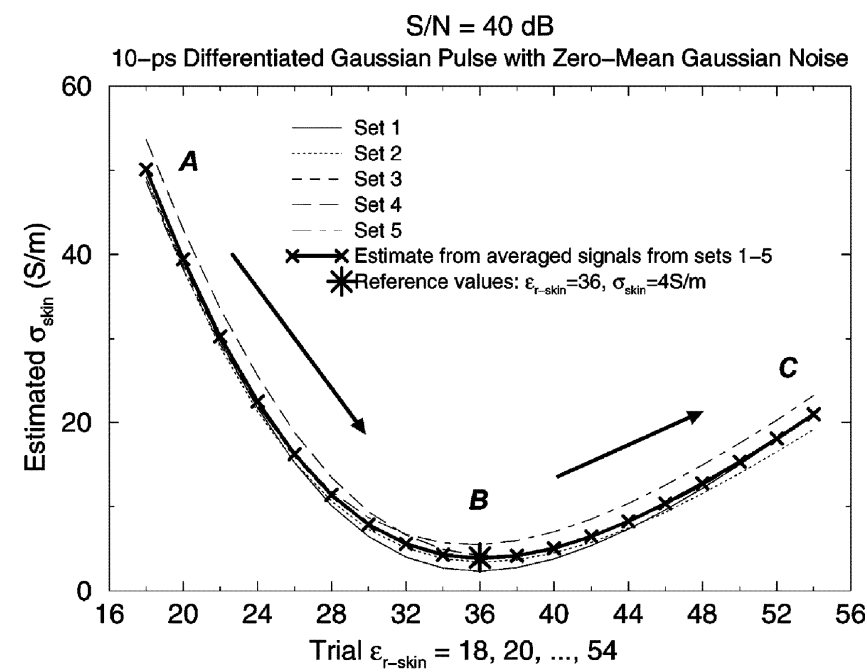

(a)

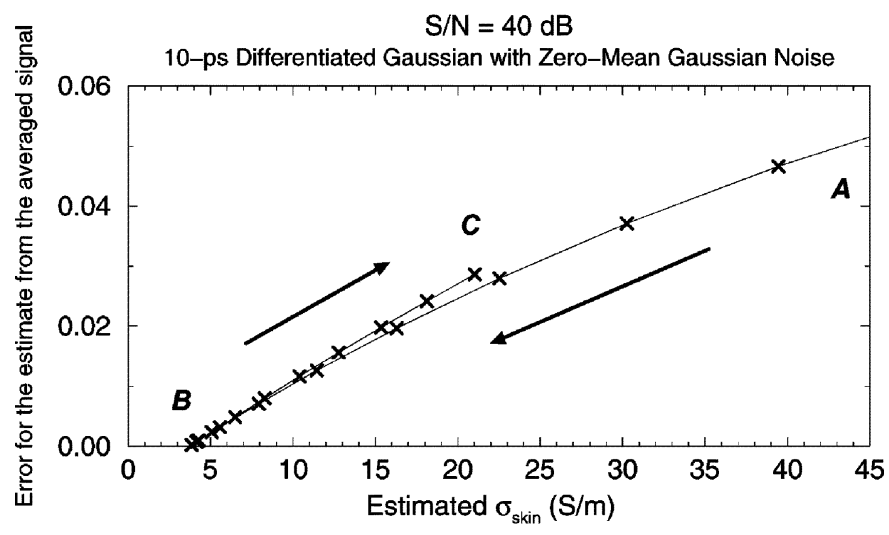

(b)

Fig. 8. (a) Sample trajectories in the $\left(\varepsilon_{r-s k i n}, \sigma_{\text {skin }}\right)$ space for the 10-ps differentiated Gaussian pulse excitation with $\mathrm{S} / \mathrm{N}=40 \mathrm{~dB}$. (b) Error versus estimated $\sigma_{\text {skin }}$ values for the averaged-backscattered-waveform case.

$\sigma$ is the conductivity ( $\mathrm{S} / \mathrm{m})$; and $\varepsilon=\varepsilon_{0} \varepsilon_{r}$ is the electrical permittivity $(\mathrm{F} / \mathrm{m})$, the product of relative permittivity $\varepsilon_{r}$ with the free-space permittivity $\varepsilon_{0}=8.854 \times 10^{-12} \mathrm{~F} / \mathrm{m}$.

In two dimensions and in the absence of independent current sources, (1) can be reduced to the TM-mode scalar equation involving Cartesian components of the field vectors

$$
\frac{\partial H_{y}}{\partial x}-\frac{\partial H_{x}}{\partial y}=\sigma E_{z}+\varepsilon \cdot \frac{\partial E_{z}}{\partial t} .
$$

This equation governs both the measured backscattered signal and the FDTD-computed trial backscattered signal

$$
\begin{gathered}
\frac{\partial H_{y, M}}{\partial x}-\frac{\partial H_{x, M}}{\partial y}=\sigma_{M} E_{z, M}+\varepsilon_{M} \cdot \frac{\partial E_{z, M}}{\partial t} \\
\frac{\partial H_{y, T}}{\partial x}-\frac{\partial H_{x, T}}{\partial y}=\sigma_{T} E_{z, T}+\varepsilon_{T} \cdot \frac{\partial E_{z, T}}{\partial t}
\end{gathered}
$$

where the subscript $M$ refers to measured signal variables and the subscript $T$ refers to trial signal variables. The excitation for both the measured and the trial pulse is the same and a linear function of time

$$
E_{z, M}=E_{z, T}=a t+b
$$




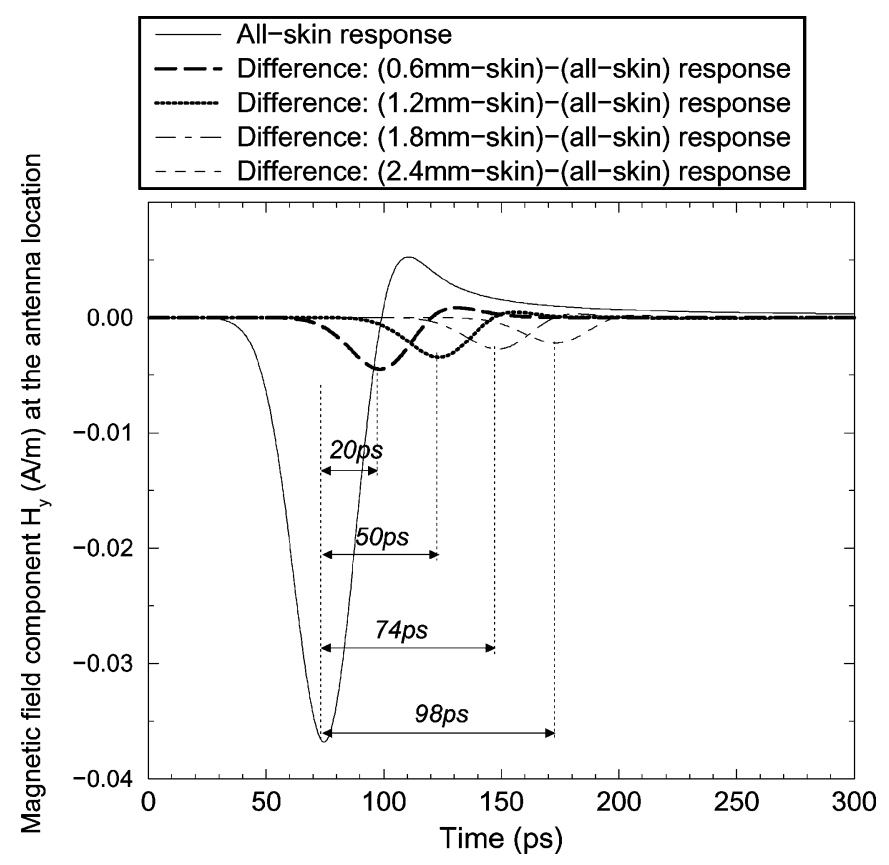

Fig. 9. Waveforms illustrating a strategy for estimating the skin thickness making use of the previously determined values of $\varepsilon_{r \text {-skin }}$ and $\sigma_{\text {skin }}$. We compare the simulated measured response (magnetic field component at the location of the antenna) for four finite skin-thickness cases with a simulation which assumes a homogeneous skin half-space.

and

$$
\frac{\partial E_{z, M}}{\partial t}=\frac{\partial E_{z, T}}{\partial t}=a
$$

where $a$ and $b$ are constants with appropriate units.

The proposed inverse-scattering iterative scheme computes the error based on energy difference. In essence, the iterative process attempts to minimize the following difference:

$$
\left[\left(\frac{\partial H_{y, M}}{\partial x}-\frac{\partial H_{x, M}}{\partial y}\right)-\left(\frac{\partial H_{y, T}}{\partial x}-\frac{\partial H_{x, T}}{\partial y}\right)\right]^{2} \longrightarrow 0 .
$$

In order for this difference to reach zero, while observing (2), (4)-(6), the following condition must be fulfilled:

$$
\left[\left(\sigma_{M} \cdot(a t+b)+\varepsilon_{M} \cdot a\right)-\left(\sigma_{T} \cdot(a t+b)+\varepsilon_{T} \cdot a\right)\right]^{2}=0 .
$$

Clearly, (8) is satisfied when the trial values of parameters are equal to the measured parameter values, i.e., when $\varepsilon_{T}=\varepsilon_{M}$ and $\sigma_{T}=\sigma_{M}$. However, (8) is satisfied also for the following condition:

$$
\varepsilon_{T}=-\frac{(a t+b)}{a} \cdot \sigma_{T}+\left[\sigma_{M} \cdot \frac{(a t+b)}{a}+\varepsilon_{M}\right] .
$$

Therefore, the minimal error condition of (8) and the final (9) tells us that linear relation between $\varepsilon_{T}$ and $\sigma_{T}$ with appropriate constants is a sufficient condition for reconstruction of $\varepsilon$ and $\sigma$ parameters by the proposed inverse-scattering scheme when the excitation signal is a linear function of time. This was observed even for the noise study in the case of the the linear, 5-ps ramp signal excitation. The potential benefit of the demonstrated linearity of the generated search trajectory in the $\left(\varepsilon_{r-\text { skin }}, \sigma_{\text {skin }}\right)$ space is as follows: linear excitation can be used for two trial $\varepsilon_{r-\text { skin }}$ values, and the rest of the values along the anticipated linear $\left(\varepsilon_{r-\text { skin }}, \sigma_{\text {skin }}\right)$ trajectory can be obtained by interpolation or extrapolation. Then, error values for the sets of $\left(\varepsilon_{r-\text { skin }}\right.$, $\sigma_{\text {skin }}$ ) obtained by this means can be tested with the noise-robust 10-ps differentiated Gaussian pulse excitation. This would significantly decrease the computational cost of the dielectric recovery procedure.

\section{CONCLUSION}

This work presented investigations of a novel 2-D time-domain inverse-scattering technique for determination of near-surface material properties at microwave frequencies. The new technique is based upon the use of an FDTD forward-scattering program element embedded within a numerical feedback loop containing a nonlinear optimization routine. A systematic parameter-perturbation strategy is adopted to permit searching of a multidimensional parameter space for the electrical properties of the materials.

There are two significant findings of this work. First, the use of a time-linear (ramp) excitation yields a linear search trajectory in $\left(\varepsilon_{r}, \sigma\right)$ space. Second, the use of a short bipolar excitation signal provides a sharp null of the energy-normed error along the search trajectory even when the backscattered signal is contaminated with a significant level of additive zero-mean Gaussian noise. Proper combination of time-linear and short bipolar pulse excitation data can yield an efficient and robust search strategy.

A logical near-term extension of this work is to construct an additional software-element which would use the same technique to obtain the electrical properties of the material underlying the surface layer after first determining the layer's thickness, permittivity and conductivity. A second extension of this technique would be to exploit its potential multidimensional search capabilities to determine the frequency dispersion of the electrical properties of the surface layer and the underlying materials.

\section{REFERENCES}

[1] J. Ch. Bolomey, D. Lesselier, C. Pichot, and W. Tabbara, "Spectral and time domain approaches to some inverse scattering problems," IEEE Trans. Antennas Propagat., vol. AP-29, pp. 206-212, 1981.

[2] S. Coen, K. K. Mei, and D. J. Anelakos, "Inverse scattering technique applies to remote sensing of layered media," IEEE Trans. Antennas Propagat., vol. 29, pp. 298-306, 1981.

[3] W. Tabbara, "Reconstruction of permittivity profiles from a spectral analysis of the reflection coefficient," IEEE Trans. Antennas Propagat., vol. 27, pp. 241-244, 1979.

[4] M. Mostafavi and R. Mittra, "Remote probing of inhomogeneous media using parameter optimization techniques," Radio Sci., vol. 7, no. 12, pp. 1105-1111, 1972.

[5] C. J. Leuschen and R. G. Plumb, "A matched-filter-based reverse-time migration algorithm for ground-penetrating radar data," IEEE Trans. Geoscience and Remote Sensing, vol. 39, pp. 929-936, May 2001.

[6] J. F. Ma, W. H. Yu, and R. Mittra, "Detection of buried dielectric cavities using the finite-difference time-domain method in conjunction with signal processing techniques," IEEE Trans. Antennas Propagat., vol. 48, pp. 1289-1294, Sept. 2000.

[7] A. Qing, C. K. Lee, and L. Jen, "Electromagnetic inverse scattering of two-dimensional perfectly conducting objects by real-coded genetic algorithm," IEEE Trans. Geoscience and Remote Sensing, vol. 39, pp. 665-676, Mar. 2001.

[8] K. R. Umashankar, S. Chaudhuri, and A. Taflove, "Finite-difference time-domain formulation of an inverse scattering scheme for remote sensing of inhomogeneous lossy layered media," J. Electromagn. Waves and Applicat., vol. 8, pp. 489-509, 1994. 
[9] M. A. Strickel, A. Taflove, and K. R. Umashankar, "Finite-difference time-domain formulation of an inverse scattering scheme for remote sensing of conducting and dielectric targets," J. Electromagn. Waves and Applicat., vol. 8, pp. 510-529, 1994.

[10] C. Gabriel, S. Gabriel, and E. Corthout, "The dielectric properties of biological tissues: I. Literature survey," Phys. Medicine and Biol., vol. 41, no. 11, pp. 2231-2249, Nov. 1996.

[11] S. Gabriel, R. W. Lau, and C. Gabriel, "The dielectric properties of biological tissues: II. Measurements on the frequency range $10 \mathrm{~Hz}$ to 20 GHz," Phys. Medicine and Biology, vol. 41, no. 11, pp. 2251-2269, Nov. 1996.

[12] — "The dielectric properties of biological tissues: III. Parametric models for the dielectric spectrum of tissues," Phys. Medicine and Biolo., vol. 41, no. 11, pp. 2271-2293, Nov. 1996.

[13] J. P. Grant, R. N. Clarke, G. T. Symm, and N. M. Spyrou, "In vivo dielectric properties of human skin from $50 \mathrm{mhz}$ to $2.0 \mathrm{ghz}$," Phys. Medicine and Biol., vol. 33, no. 5, pp. 607-612, 1988.

[14] M. Olenius and O. Johansson, "Variations in epidermal thickness in expanded human breast skin," Scandinavian J. Plastic and Reconstructive and Hand Surgery, vol. 29, pp. 15-20, 1995.

[15] T. L. Pope Jr., M. E. Read, T. Medsker, A. J. Buschi, and A. N. Brenbridge, "Breast skin thickness: Normal range and causes of thickening shown on film-screen mammography," J. Canadian Assoc. Radiologists, vol. 35, pp. 365-368, Dec. 1984.

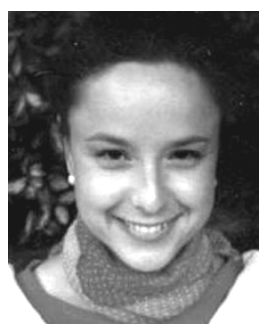

Milica Popović (M'94) received the B.Sc.degree in electrical engineering from the University of Colorado at Boulder, in 1994 and the M.Sc. and Ph.D. degree from Northwestern University, Evanston, IL, in 1997 and 2001, respectively.

Since 2001, she has held a position of Assistant Professor with the Department of Electrical and Computer Engineering, McGill University, Montréal, Canada. She is involved in improvement of teaching electromagnetics as a key and fundamental subject of the electrical engineering curriculum. Her research interests include methods of numerical electromagnetics, with the emphasis on biomedical applications, and antenna theory and design.

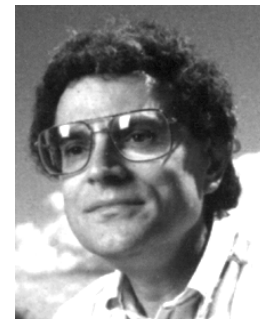

Allen Taflove (F'90) was born in Chicago, IL, on June 14, 1949. He received the B.S., M.S., and Ph.D. degrees in electrical engineering from Northwestern University, Evanston, IL, in 1971, 1972, and 1975, respectively.

After nine years as a Research Engineer at the IIT Research Institute, Chicago, IL, he returned to Northwestern in 1984. Since 1988, he has been a Professor in the Department of Electrical and Computer Engineering, McCormick School of Engineering, Northwestern. Since 1972, he has pioneered basic theoretical approaches and engineering applications of finite-difference time-domain (FDTD) computational electrodynamics. He coined the FDTD acronym in a 1980 IEEE paper, and in 1990 was the first person to be named a Fellow of IEEE in the FDTD area. Currently, FDTD is one of the most powerful and widely used methods for solving Maxwell's equations to model linear and nonlinear electromagnetic wave interactions with electrically large and complex structures. In 1995, he authored a popular textbook on this subject Computational Electrodynamics: The Finite-Difference Time-Domain Method (Boston, MA: Artech House, 1995), which is now in its second edition (2000), with a third edition is planned for 2005. In total, he has authored or co-authored four books, 14 invited papers or chapters in books, 79 refereed journal papers, approximately 200 conference papers and abstracts, and 14 U.S. patents. These publications resulted in his being named to the Highly Cited Researchers list of the Institute for Scientific Information. He has been the thesis adviser of $16 \mathrm{Ph} . \mathrm{D}$. recipients who hold professorial or technical staff positions at major institutions including research universities and national labs. Currently, he serves as the faculty Master of Northwestern's 140-student Slivka Residential College of Science and Engineering. He also serves as the Faculty Advisor to Northwestern's Undergraduate Design Competition, the student chapters of the Eta Kappa Nu and Tau Beta Pi engineering honor societies, and McCormick's Honors Program in Undergraduate Research. His efforts on behalf of students at all levels were recognized by Northwestern when he was named a C. D. McCormick Professor of Teaching Excellence in 2000. His research interests span much of the electromagnetic spectrum. He and his students are currently modeling electrodynamic phenomena ranging from geophysically induced extremely low frequency wave propagation about the entire Earth to the lasing behavior of aggregates of micron-scale zinc-oxide particles exhibiting four-level quantum-system characteristics. The principle that "Maxwell's equations work from dc to light" is vividly demonstrated in his laboratory every day. 